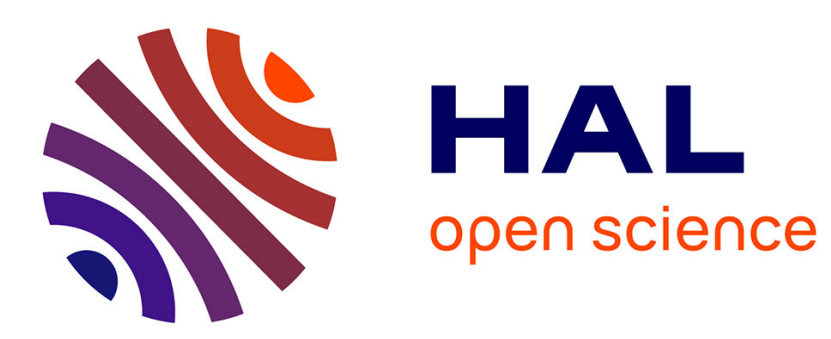

\title{
Dielectric and elastic-response investigations of polar motion in potassium-sodium tantalate
}

\author{
Mario Maglione, J. Joffrin, U.T. Höchli, J. Pelous
}

\section{To cite this version:}

Mario Maglione, J. Joffrin, U.T. Höchli, J. Pelous. Dielectric and elastic-response investigations of polar motion in potassium-sodium tantalate. Journal de Physique, 1988, 49 (6), pp.959-965. 10.1051/jphys:01988004906095900 . jpa-00210783

\section{HAL Id: jpa-00210783 https://hal.science/jpa-00210783}

Submitted on 1 Jan 1988

HAL is a multi-disciplinary open access archive for the deposit and dissemination of scientific research documents, whether they are published or not. The documents may come from teaching and research institutions in France or abroad, or from public or private research centers.
L'archive ouverte pluridisciplinaire HAL, est destinée au dépôt et à la diffusion de documents scientifiques de niveau recherche, publiés ou non, émanant des établissements d'enseignement et de recherche français ou étrangers, des laboratoires publics ou privés. 
Classification

Physics Abstracts

$62.65-77.40-64.70 \mathrm{~K}-64.70 \mathrm{P}$

\title{
Dielectric and elastic-response investigations of polar motion in potassium-sodium tantalate
}

\author{
M. Maglione $\left({ }^{1, *}\right)$, J. Joffrin $\left({ }^{2}\right)$, U. T. Höchli $\left({ }^{1}\right)$ and J. Pelous $\left({ }^{3}\right)$ \\ ( ${ }^{1}$ ) Institut de Physique Expérimentale, EPFL, 1015 Lausanne, Switzerland and IBM Research, Zurich \\ Research Laboratory, Säumerstr. 4, 8803 Rüschlikon, Switzerland \\ (2) Laboratoire de Physique des Solides, Université d'Orsay, 91405 Orsay, France \\ $\left({ }^{3}\right)$ Laboratoire de Science des Matériaux Vitreux, Place Eugène Bataillon, 34060 Montpellier Cedex, France
}

(Reçu le 18 décembre 1987, accepté le 23 février 1988)

\begin{abstract}
Résumé. - L'instabilité polaire de $\mathrm{K}_{1-x} \mathrm{Na}_{x} \mathrm{TaO}_{3}$ est associée à une variation critique des réponses linéaire et non linéaire. Les résultats sur les susceptibilités diélectriques et élastiques montrent un ralentissement critique de $10^{9}$ à $10^{7} \mathrm{~Hz}$ de l'oscillation des ions $\mathrm{Ta}^{5+}$ en s'approchant de la température critique. La polydispersion de la susceptibilité à basses fréquences et la divergence de la susceptibilité non linéaire sont des indications en faveur du gel d'une configuration désordonnée à une température légèrement inférieure à la température critique (extrapolée). Les modèles habituels des verres dilués n'expliquent pas cette quasi-coïncidence. Par contre, nous cherchons un scénario dans lequel les interactions de type ferroélectrique et les interactions frustrées entre les impuretés dipolaires sont accrues par la corrélation entre les distorsions dipolaires du réseau.

Abstract. - The polar instability of $\mathrm{K}_{1-x} \mathrm{Na}_{x} \mathrm{TaO}_{3}$ is associated to critical linear and nonlinear phenomena. Data on linear dielectric and elastic susceptibilities show a critical slowing down from $10^{9}$ to $10^{7} \mathrm{~Hz}$ of the $\mathrm{Ta}^{5+}$ motion on approaching the critical temperature. Polydispersive susceptibility at low frequency and critical nonlinearity are evidence for freezing of a disordered configuration only slightly below the (extrapolated) critical temperature. Standard models of dilute glasses do not account for this near-coincidence. Instead, we search for a scenario in which both the ferroelectric and the frustrated interactions between impurity dipoles are enhanced by the critical correlation of lattice dipolar displacements.
\end{abstract}

\section{Introduction.}

Solid solutions of perovskite oxides are abundant [1] and of scientific [2] and technical interest [3]. Few of them have been thoroughly investigated, and the ones that have been show quite complex behaviour $[4,5]$. Of the 16 simple perovskite oxides, the majority have a polar instability [1]. In particular $\mathrm{KTaO}_{3}$, the host lattice of the crystals reported here, has a dielectric susceptibility which reaches $5 \times 10^{3}$ at $4 \mathrm{~K}$. Associated to large susceptibilities is an enhanced correlation of polar motion [6]. Accordingly, in $\mathrm{KTaO}_{3}$, impurities interact at larger distances [7] than in inert lattices like $\mathrm{NaCl}$. The interaction between impurities in polarizable lattices has been the subject of a perturbation calculation [7]

(*) Permanent address : Laboratoire de Physique du Solide, Université de Bourgogne, B.P. 138, 21004 Dijon Cedex, France. and computer simulation [8]. Both approaches showed strong deviations from the familiar dipolar form when the impürities were closer than some critical distance $r_{\mathrm{c}}$. The complexity of this interaction $J_{i j}$ between dipoles $p_{i}$ and $p_{j}$ and its long range preclude a theoretical treatment of the Hamiltonian

$$
\mathscr{H}=\sum J_{i j} p_{i} p_{j}
$$

In absence of reliable theoretical predictions, we attempt to gain experimental insight into the material in which such interactions between impurities in a polarizable lattice play a dominant role.

The system $\mathrm{K}_{1-x} \mathrm{Na}_{x} \mathrm{TaO}_{3}(\mathrm{KNT})$ is a mixed perovskite oxide. It has been the subject of several studies [9-11] which attributed its polar phase the character of an ordered state. Some observations, however, are incompatible with the ferroelectric-like ordered nature of the low-temperature phase of $\mathrm{KNT}$ : There are no domain walls visible $[12,13]$ as 
in isostructural ferroelectric $\mathrm{BaTiO}_{3}$, and the polarization cannot be switched [13] by electric fields $E$ at temperatures $T$ below the critical $T_{\mathrm{c}}$. A search for the critical nuclear magnetic relaxation $T_{1}$ of ${ }^{23} \mathrm{Na}$ resulted in a report that $T_{1}$ was in fact continuous right through $T_{\mathrm{c}}$ [14]. The ensuing uncertainty regarding the nature of the transition leaving local properties unaffected prompted a re-investigation of collective phenomena [15] in KNT and associated compounds [16]. The main result was the discovery of two relaxation contributions. A high-frequency relaxation peak showed monodispersive character, independent of $\mathrm{Na}$ and $\mathrm{Nb}$ addition [16]. This relaxation was attributed to ordered Ta motion. A low-frequency relaxation phenomenon showed polydispersive character, dependent on $\mathrm{Na}$ content, and was associated to a glass transition. Both phenomena were observed in the same sample $(x=0.2)$ at neighbouring temperatures.

In this paper, we wish to present a completed investigation of dielectric relaxation and nonlinear susceptibility in five samples with $\mathrm{Na}$ content $x$ ranging from 0.08 to 0.24 . The data are supplemented by acoustic-resonance and Brillouin scattering measurements. The purpose of this paper is to correlate the seemingly independent order and disorder phenomena. In particular, we shall present clear evidence that nonlinear susceptibility is critical in the sense of a glass transition [17]. We point out that critical linear and nonlinear susceptibilities are related in the $x-T$ phase diagram. This relation calls for a common origin for the two phenomena which we shall propose at the end.

\section{Experimental results.}

A previous investigation on the nonlinear dielectric susceptibility in $\mathrm{K}_{1-x} \mathrm{Na}_{x} \mathrm{TaO}_{3}$ concentrated on the sample with $x=0.2[15]$. While it established the existence of a glass phase, it did not attempt to dwell on the origin of the interactions leading to a glassy state. In this section, we expand the previous investigation to samples with different $\mathrm{Na}$ concentrations. Furthermore, we present dynamical results on the elastic compliance on the same sample, and finally we intend to correlate the different results in a qualitative way.

2.1 STATIC NONLINEAR SUSCEPTIBILITY. - We present the coefficient $\varepsilon_{\mathrm{NL}}$, called nonlinear susceptibility and defined by

$$
\varepsilon_{\mathrm{s}}(E, T)=\varepsilon_{\mathrm{s}}(0, T)-\varepsilon_{\mathrm{NL}}(T) E^{2}+(O)\left(E^{4}\right) .
$$

Here $\varepsilon_{\mathrm{s}}$ denotes the static susceptibility, measured in fact at $f=1 \mathrm{kHz}$ to obtain optimum precision. The inability to measure $\varepsilon_{\mathrm{s}}$ directly forces us to exclude data obtained in a low-frequency dispersive regime. These data fall into a temperature range around $T_{\mathrm{g}}$. Lowering the measuring frequency to, say, $10 \mathrm{~Hz}$ would be of little help in approaching $T_{\mathrm{g}}$ in view of the slow algebraic dispersion [15] of the imaginary part of the dielectric susceptibility $\varepsilon_{2} \sim \omega^{\nu}, \nu \sim 0.1$, there. Data of $\varepsilon_{\mathrm{s}}(E, T)$ have been fitted to equation (2) in a standard way [18]. The result for $\varepsilon_{\mathrm{NL}}$ is plotted in figure 1 as a function of temperature for five different samples. The analysis in terms of the scaling function [18] $a(T)=\varepsilon_{\mathrm{NL}}(T) / T \varepsilon_{\text {lin }}^{4}$ yields the dashed curves also shown in the figure. According to this analysis, which is identical to that of [15] and [18], a divergence of $a(T)$ of the form $a \sim\left(T-T_{\mathrm{g}}\right)^{-\gamma}$ indicates a transition to a glassy state provided $T_{\mathrm{g}}>0$. Inspection of figure 1 indicates that $T_{\mathrm{g}}<0$ for $8 \%$ and $\sim 0$ for $12 \%$. We find $T_{\mathrm{g}}=5$ and $11 \mathrm{~K}$ for $16 \%$ and $20 \% \mathrm{Na}$ doping, respectively. No divergence of $a(T)$ is indicated for $24 \% \mathrm{Na}$ doping.

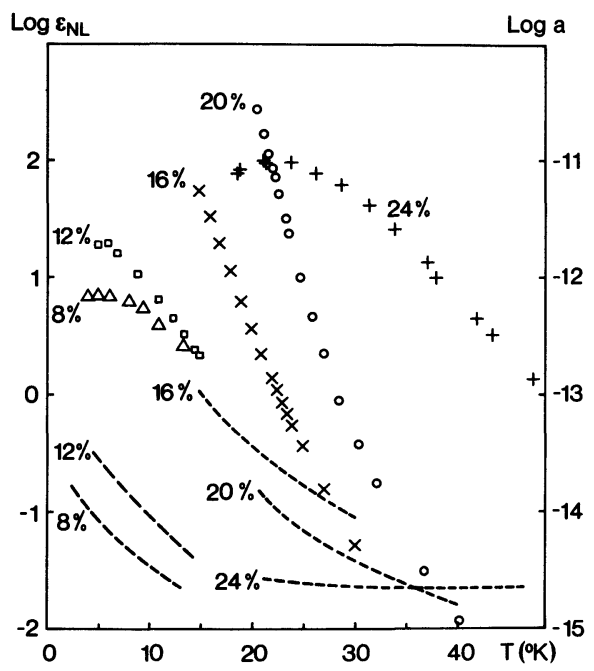

Fig. 1. - Nonlinear dielectric susceptibility data $\partial \varepsilon / \partial\left(E^{2}\right)$, scaling functions $a(T)=$ const. $\varepsilon_{\mathrm{NL}} /\left(T \varepsilon^{4}\right)$ vs. temperature fitted to $\left[\left(T-T_{\mathrm{g}}\right) / T\right]^{-\gamma}$. The samples are those used in an earlier investigation [11].

2.2 DieleCtRIC DISPERSION. - At sufficiently low temperatures, the samples with $16 \% \mathrm{Na}$ and $20 \% \mathrm{Na}$ reported earlier [15] are dispersive. The results of $\varepsilon_{2}(\omega)$ for $16 \% \mathrm{Na}$ doping are plotted on $\log -\log$ scale in figure 2 . The fit to straight lines indicates that $\varepsilon_{2}=A \omega^{\nu}$, and the parameters are shown in figure 3 . We note that below about $15 \mathrm{~K}$ the amount of dispersion given by $A$ rises sharply. In this temperature range, measurement of the dynamical nonlinear dispersion [19] is a nontrivial matter not attempted here. Other samples show only negligible low-frequency dispersion.

2.3 Elastic COMPLianCE. - A small electric bias (of $10^{3} \mathrm{~V} / \mathrm{m}$ ) is sufficient to render $\mathrm{KTaO}_{3}$ piezoelectric. Such samples resonate at a frequency $f_{\mathrm{r}}$ [20]. Real and imaginary parts of the elastic compliance 


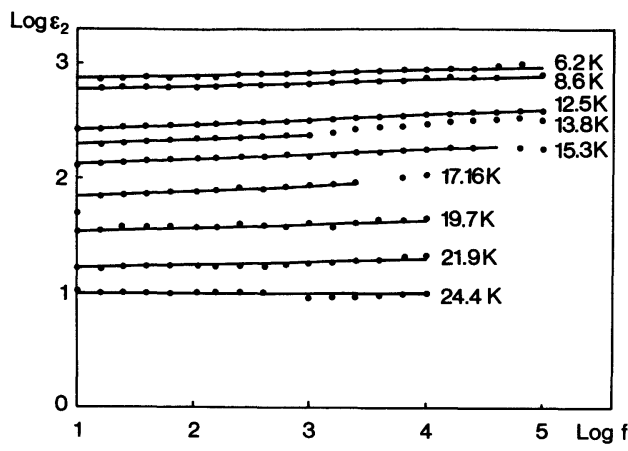

Fig. 2. - Imaginary part of the audio-frequency dielectric susceptibility vs. $\omega$ in $\log$-log representation. Straight lines : $\varepsilon_{2}=A(T) \omega^{\nu(\mathrm{T})}$.

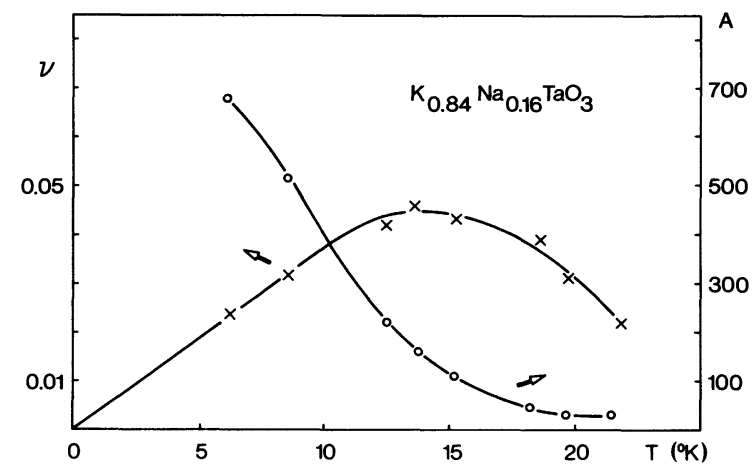

Fig. 3. - Parameters $A$ and $\nu$ vs. temperature (see Fig. 1 or Eq. (1)). Note that $\nu$ is maximum at $T_{\mathrm{g}}$ and $A$ vanishes above $T_{\mathrm{g}}$.

are obtained from the resonance frequencies $f_{\infty}$ (far away from dispersion regimes) and $f_{\mathrm{r}}$ (in the dispersion regime) and from their width $\Delta f_{\mathrm{r}}$ as

$$
\begin{gathered}
\operatorname{Re}(s)-s_{\infty}=\left(f_{\infty}^{-2}-f_{\mathrm{r}}^{-2}\right)\left(4 l^{2} \rho\right)^{-1} \\
\operatorname{Im}(s)=\Delta f_{\mathrm{r}} f_{\mathrm{r}}^{-1}\left(f_{\infty}^{-2}-f_{\mathrm{r}}^{-2}\right)\left(4 l^{2} \rho\right)^{-1} .
\end{gathered}
$$

Here $s_{\infty}$ is the elastic compliance $s_{11}$ above the dispersion regime, $l$ and $\rho$ are the length $(5.9 \mathrm{~mm})$ and the mass density $\left(7000 \mathrm{~kg} / \mathrm{m}^{3}\right)$ of the sample. Equations (3) and (4) hold for a long thin sample vibrating in length extension, $l$ being parallel to the $x$-axis of the crystal. We plot the real part of the critical elastic compliance vs. $T$ for $x=0.2$ in figure 4. It shows the same smeared-out step function as found previously [11] and an $x$-dependent step for all other samples with $x \gg 0.12$. Evaluation of the resonance data in terms of a Debye-type monodispersion yields

$$
s_{\infty}-s=\Delta s /(1+j \omega \tau),
$$

where $\tau$ is the relaxation time of the strain. We have plotted the relaxation time of the local quadrupoles [21], $t_{\mathrm{q}}^{-1}=6 \tau^{-1}$, vs. temperature in figure 5 . We find $10^{7} \mathrm{~s}^{-1}<\tau^{-1}<2 \times 10^{8} \mathrm{~s}^{-1}$. On lowering $T$,

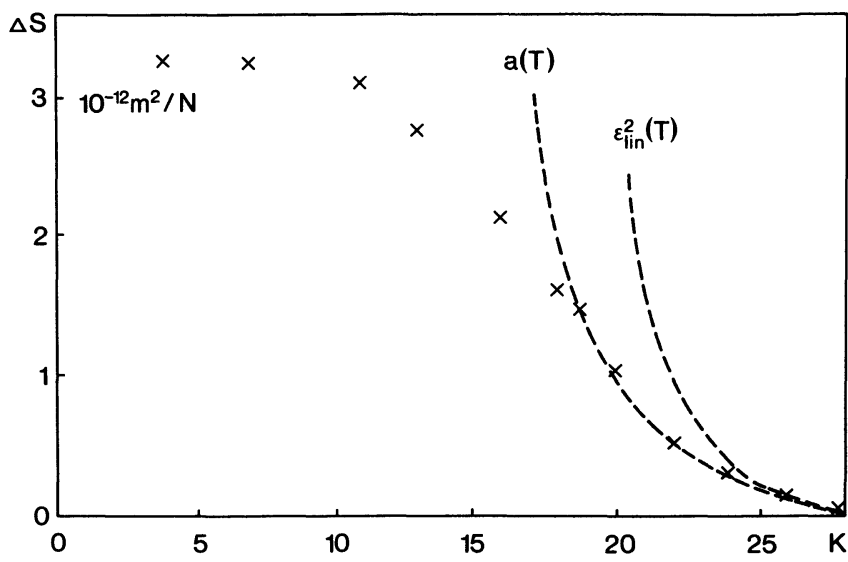

Fig. 4. - Elastic compliance of $\mathrm{K}_{0.8} \mathrm{Na}_{0.2} \mathrm{TaO}_{3}$ vs. temperature. For comparison, scaling function $a(T)$ (see Fig. 1) and square of linear susceptibility adjusted to scale at $25 \mathrm{~K}$.

$\tau^{-1}$ drops to its lowest value of $10^{7} \mathrm{~s}^{-1}$ at $\sim 13 \mathrm{~K}$, and below $13 \mathrm{~K}$ it stays constant.

We have also investigated acoustic relaxation in presence of a field: in figure 6 we plot $\tau_{q}^{-1}$ vs.

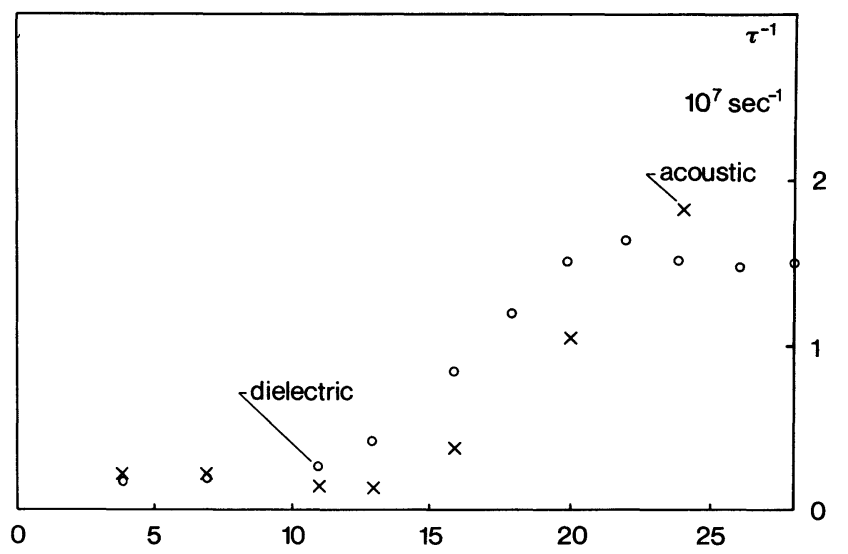

Fig. 5. - Dielectric and acoustic relaxation rates vs. temperature, $\mathrm{K}_{0.8} \mathrm{Na}_{0.2} \mathrm{TaO}_{3}$.

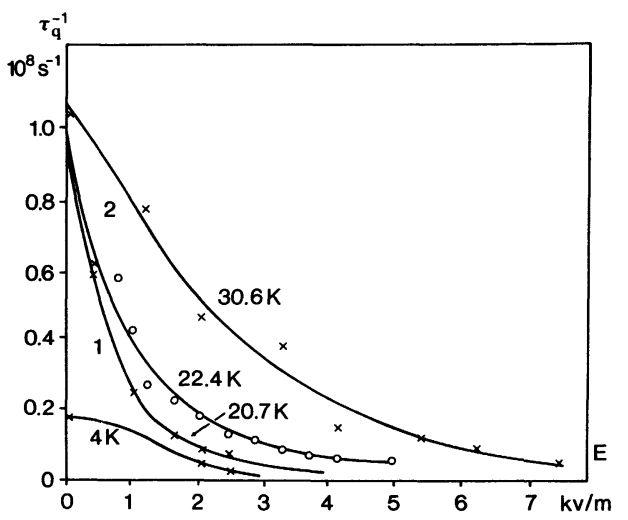

Fig. 6. - Acoustic and dielectric relaxation rates vs. field, $\mathrm{K}_{0.8} \mathrm{Na}_{0.2} \mathrm{TaO}_{3}$. 
$E$ at different temperatures, again for $x=0.20$. Moderate fields of a few $\mathrm{kV} / \mathrm{m}$ are able to slow down relaxation by an order of magnitude.

2.4 BRILLOUIN SCATTERING. - Measurements were performed using the $514.5 \mathrm{~nm}$ line of a singlemode Argon laser and a double-pass Fabry-Pérot interferometer with a free spectral range of $60 \mathrm{GHz}$. The right-angle scattering configuration was used; the incident and scattered beams were perpendicular to the [100] faces of the samples, so that the acoustic phonons selected were propagating along the (110) direction. The polarization of the incident light was normal to the plane of scattering. Two phonon peaks were attributed to a longitudinal and a shear mode ; their elastic constants are $1 / 2\left(C_{11}+C_{12}+2 C_{44}\right)$ and $C_{44}$, respectively.

The variations of the frequency shifts versus temperature are plotted in figure 7 . The accuracy of the measurements is about $1 \%$. We note that the Brillouin shifts vary by less than $\sim 2 \%$ with temperature, even at $T_{\mathrm{g}}$. Thus, the relaxation rates must be below $\nu_{\mathrm{L}} / \sqrt{0.02} \sim 5 \times 10^{9} \mathrm{~Hz}$, in agreement with elastic data.

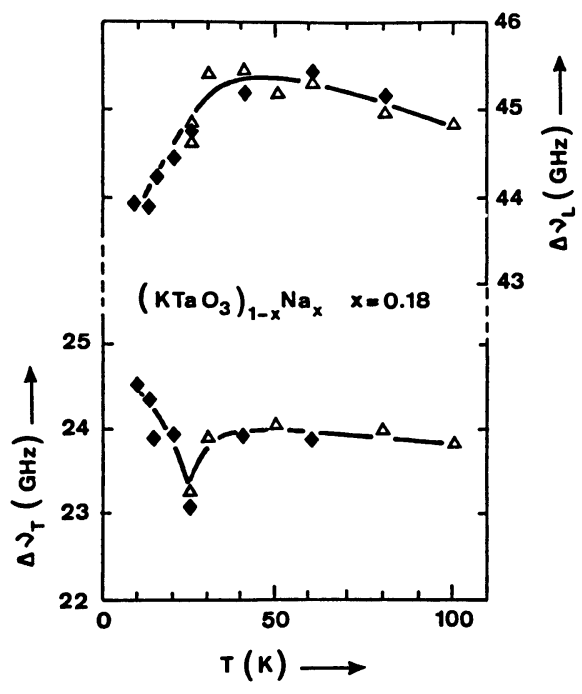

Fig. 7. - Brillouin shift due to longitudinal (upper curve) and transverse (lower curve) acoustic waves while lowering $(\triangle)$ and increasing $(\diamond) T$.

2.5 SUMMARY OF EXPERIMENTAL RESULTS. - Critical nonlinear susceptibility and algebraic dispersion of the linear susceptibility are associated to a glass transition [17], whereas critical linear susceptibility and Debye dispersion arise in a ferroelectric-type order-disorder transition [2]. Thus, not all our observations have a common origin, yet they occur under identical conditions, namely for $x>x_{\mathrm{c}}$ and near the ( $x$-dependent) $T_{\mathrm{g}}$. In the following, we attempt to explain these puzzling features.

\section{Interpretation.}

In this section, we wish to describe the critical behaviour of the susceptibility and compliance in terms of models which have been advanced in connection with phase transformations.

We recall that the linear dielectric susceptibility follows a Curie-Weiss type behavior

$$
\varepsilon_{\text {lin }} \sim\left(T-T_{\mathrm{c}}\right)^{-\gamma},
$$

where $T_{\mathrm{c}}$ depends on concentration as $T_{\mathrm{c}}=A\left(x-x_{\mathrm{c}}\right)^{1 / 2}, x_{\mathrm{c}}=0.12, A=60$. The exponent $\gamma$ depends on $T_{\mathrm{c}}$ (and thus on $x$ ); for $T_{\mathrm{c}}=0$ it approaches the value of 2 , and for larger $T_{\mathrm{c}}$ it takes the Curie-Weiss limit $\gamma=1$. In the most thoroughly investigated sample, $x=0.2, T_{\mathrm{c}}=11 \mathrm{~K}, \gamma=1.4$. The rise of the exponent $\gamma[10]$ is attributed to quantum-statistical fluctuations of the polarization arising from soft modes with frequencies $\omega>k T_{\mathrm{c}} / \hbar$ $[22,23]$. Accordingly, one expects the onset of a spontaneous macroscopic polarization below $T_{\mathrm{c}}$, a divergence of the susceptibility on both sides of $T_{\mathrm{c}}$ and a nonlinear susceptibility $\varepsilon_{\mathrm{NL}} \sim \varepsilon_{\text {lin }}^{4}$. Experiments show that no macroscopic polarization arises spontaneously; $\varepsilon_{\text {lin }}$ does not diverge below $T_{\mathrm{c}}$ [15], but the renormalized nonlinear susceptibility $\varepsilon_{\mathrm{NL}} / \varepsilon_{\text {lin }}^{4}$ does diverge. These findings are contrary to expectations from ferroelectric models, but exactly analogous results have been reported in spin glasses [17] and explained in terms of the model by Sherrington and Kirkpatrick (SK) [24]. They assume that the interaction $J_{i j}$ between species $i$ and $j$ is a random variable with a variance $\left\{\sum_{i, j}\left(J_{i j}-\bar{J}_{i j}\right)^{2}\right\}^{1 / 2}$ exceeding its average $J_{0}=\bar{J}_{i j}$.

The only random variables in KNT are the dipolar interactions between random-site off-center $[25,26]$ $\mathrm{Na}$ ions at $\mathrm{K}$ sites. It is natural to identify them as $J_{i j}$ of the SK model (Eq. (1)).

We start by estimating the polar displacements and their correlations of $\mathrm{Ta}$ and $\mathrm{Na}$ dipolar orientations in KNT. From the remanent polarization [13], $P=12 \mathrm{mc} / \mathrm{m}^{2}$, it follows that $p_{\mathrm{Ta}} \sim 7.7 \times$ $10^{-31} \mathrm{Cm}$, which corresponds to a displacement $\delta_{\mathrm{Ta}} \sim 0.01 \AA$. This compares to $\delta_{\mathrm{Na}} \sim 0.04 \AA$ as obtained from magnetic-resonance data [26] on the nuclear spin of ${ }^{23} \mathrm{Na}$ in KNT. In view of the dilution and the lesser charge, the contribution of $\mathrm{Na}$ ions to $P$ can be neglected, and it follows that the direct interaction between random-site $\mathrm{Na}$ dipoles must be much weaker than the $\mathrm{Ta}-\mathrm{Ta}$ interaction. Yet, the $\mathrm{Na}$ dipoles appear to determine the disordered character of KNT below $T_{\mathrm{c}}$. The correlation length $\xi$ of the fluctuating dipoles may be estimated from the static susceptibility. If coherent regions of size $\xi^{3}=n \times d^{3} \quad(n=$ number of dipoles, $d=$ lattice constant) are considered independent of one another, then the fluctuations of their dipole mo- 
ments $n p_{\mathrm{Ta}}$ will give rise to a susceptibility of the order of

$$
\varepsilon=(N / n)\left(n p_{\mathrm{Ta}}\right)^{2} / 3 k T \varepsilon_{0} .
$$

Here, $N$ is the total number of dipoles, $N / n$ the number of coherent regions, and $\varepsilon_{0}=8.85 \mathrm{C} / \mathrm{Vm}$ the conversion factor in SI units. We find $n=1.5 \times 10^{5}$ and $\xi \sim 200 \AA$ for $x=0.2$. This is several orders of magnitude smaller than the domain size in isostructural ferroelectric $\mathrm{BaTiO}_{3}$.

According to random-interaction models, the nonlinear susceptibility $\varepsilon_{\mathrm{NL}}$ should diverge more strongly than $\varepsilon_{\text {lin }}^{4}[18]$. The function $a(T) \equiv \varepsilon_{\mathrm{NL}} / T \varepsilon_{\text {lin }}^{4} \sim\left(T-T_{\mathrm{g}}\right)^{-\gamma_{\mathrm{NL}}}$, which is a measure for the divergence of the spin-glass susceptibility [17], is plotted in figure 4 . We find $\gamma_{\mathrm{NL}}=1.5$, 2.0 and 1.7 for $x=0.12,0.16$ and 0.2 , respectively. These findings are analogous to those on CuMn [17], and both results are compatible with the Sherrington-Kirkpatrick version of a glass. For $x=0.08$, the divergence of $a(T)$ falls into the negative temperature range. We may call this sample an incipient glass in analogy to the incipient ferroelectric. No divergence of $a(T)$ is found for $x=0.24$. This sample, which shows a very broad maximum of $\varepsilon_{\text {lin }}$ with respect to $T$, may have compositional inhomogeneities on a macroscopic scale.

From a dynamical treatment of the SK model, it was concluded [27] that the polarization decayed as $P \sim t^{-\alpha}$, and accordingly the imaginary part of the dielectric susceptibility behaved as $\varepsilon_{2}=A \omega^{\nu}$, with $A$ nonzero below $T_{\mathrm{g}}$ and $\nu \sim 1 / 2-\left(T-T_{\mathrm{g}}\right) / \pi T_{\mathrm{g}}$. As for the sample with $x=0.2$ [15], this behaviour was tested for $x=0.16$ (see Fig. 2). Again, it appears that $\nu$ is overestimated, a fact attributed to the use of mean-field theory [28]. The samples with 0.24 and 0.12 are only weakly dispersive and not suited for a quantitative analysis. No dispersion is reported for $x \leqslant 0.08$.

The critical elastic compliance originates from the coupling of the strain to the polarization. In nonpiezoelectric perovskite structure, this coupling [29] is of the form $S \times P^{2}, S$ being the strain. Accordingly, the elastic compliance gives a measure of the correlation of four polar units. Are these units the ordered $\mathrm{Ta}$ dipoles or the disordered $\mathrm{Na}$ dipoles? Coupling between ordered dipoles leads to the relation $s \sim \varepsilon_{\text {lin }}^{2}$ when treated in mean-field theory [29]. We have plotted $\varepsilon_{\text {lin }}^{2}$ vs. $T$ in figure 4 , adjusting the scale at $25 \mathrm{~K}$. The fit to $s \sim \varepsilon_{\text {lin }}^{2}$ is not convincing. We also note that in distinction to ordered $\mathrm{SrTiO}_{3}[30]$, at fit of $s(T)$ to $\left|T-T_{\mathrm{c}}\right|^{-\mu}$ on both sides of $T_{\mathrm{c}}$ is not possible. Thus, there are no critical fluctuations of the local quadrupole moments near $T_{\mathrm{c}}$. Correlation between four disordered dipoles is described by the scaling function $a(T)$. This function (Fig. 4) does not fit the data of $s(T)$ either.
While static scaling arguments fail to elucidate the origin of the elastic anomaly, considerations on the dynamical behavior are more suggestive. In the critical temperature range, $\varepsilon_{\text {lin }}$ is dispersive as $(1+j \omega \tau)^{-1}$, i.e., of Debye type for $\omega \geq 10^{5} \mathrm{~Hz}$, and as $\omega^{\nu}$, i.e., algebraic for $\omega \leq 10^{5} \mathrm{~Hz}$. For ultrasonic dispersion, only very few data points are usually available. We concentrate on our resonance data taken at $3 \times 10^{5} \mathrm{~Hz}$ and postulate that, in analogy to the dielectric data, these data be explained by monodispersion. As a result, we find the true static value for the elastic compliance which practically coincides with the values at $3 \times 10^{5} \mathrm{~Hz}$ (Fig. 5). In addition, we determine the relaxation time of the strain $\tau_{s}^{-1}$, and by considering the channels through which relaxation can occur [20], we find $\tau_{\mathrm{q}}^{-1}=\tau_{\mathrm{s}}^{-1} / 6$, where $\mathrm{q}$ indicates a local quadrupolar distortion. The result for $\tau_{d}$ and $\tau_{\mathrm{q}}$ is plotted in figure 5 : both $\tau_{\mathrm{d}}$ and $\tau_{\mathrm{q}}$ slow down critically and have nearly the same value, $\tau^{-1} \sim$ $2 \times 10^{7} \mathrm{~s}^{-1}$ at $T_{\mathrm{g}}$. The small step in the acoustic phonons measured by Brillouin scattering at $5 \times 10^{9} \mathrm{~Hz}$ is in agreement with the value of $\tau_{\mathrm{d}}$ and $\tau_{\mathrm{q}}$ deduced from ultrasonic resonance data.

The motion of the dipoles has been attributed to $\mathrm{Ta}^{5+}$ ions earlier [16]. Since the elastic quadrupoles have the same time scale in the entire critical region, those quadrupoles and thus the elastic anomaly measured at $3 \times 10^{5} \mathrm{~Hz}$, are associated to ordered motion of $\mathrm{Ta}^{5+}$. Our data do not provide evidence for or against arithmetic elastic dispersion as predicted for spin glasses [31]. On approaching $T_{\mathrm{c}}$, we find that both the linear dielectric and elastic susceptibilities start diverging, a precursory sign for the onset of ordering. Rather than exhibiting a range of criticality (like $s \sim\left|T-T_{\mathrm{c}}\right|^{-\nu}$ ), they are both stabilized at some finite value, indicating that no longrange order is established. At about the same temperature, and in the same samples, critical nonlinearity and arithmetic dispersion occur ; these effects are attributed to a freezing-in of a disordered polar configuration. The ferroelectric interactions $\bar{J}_{j i}$ and the spin-glass interactions $\operatorname{var}\left(J_{i j}\right)$ must therefore be of the same order of magnitude. While conceptually appealing, it is not possible to attribute $\bar{J}_{i j}$ to the strong ferroelectric Ta-Ta interaction and $\operatorname{var}\left(J_{i j}\right)$ to the weak random $\mathrm{Na}-\mathrm{Na}$ interaction : instead we wish to describe a scenario of polar freezing in KNT which, admittedly, is speculative.

We start by recalling that collective polar phenomena in perovskite oxides are commonly attributed to the nonlinear polarizability of the oxygen ions [32]. This leads to attraction between Ta and $\mathrm{O}$ in linear chains. The attraction is enhanced by $\mathrm{Nb}$ substituting for $\mathrm{Ta}$, leading to a condensation of an ordered polar mode. Since $\mathrm{Na}$, substituting $\mathrm{K}$, is not at the proper position to enhance the $\mathrm{Ta}-\mathrm{O}$ 
attraction, the enhancement of polar interactions due to $\mathrm{Na}$ addition must have a different origin.

We propose that the oxygen polarizability is enhanced through structural relaxation. When $20 \% \mathrm{Na} \quad\left(r_{\mathrm{Na}}=0.95 \AA\right)$ substitute for $\mathrm{K}$ $\left(r_{\mathrm{K}}=1.34 \AA\right)$, the occupied volume per unit cell shrinks by $2.5 \%$. The total unit-cell volume, however, shrinks by only $0.75 \%[1], d(0)=3.99 \AA$, $d(0.2)=3.98 \AA$. Accordingly, more space is available for the oxygen to deform. Upon lowering the temperature or increasing $\mathrm{Na}$ content, the motion of the $\mathrm{Ta}$ becomes more and more correlated and stronger. At the same time, the coupling between Ta and off-center $\mathrm{Na}$ is enhanced. This leads to an effective interaction between $\mathrm{Na}$ dipoles which is enhanced when $x$ increases. In the language of the SK model [23], where the probability for interaction $J$ is

$$
p(J)=\exp -\left[\left(J-J_{0}\right) / \Delta J\right]^{2},
$$

both the average interaction $J_{0}$ and its variance $\Delta J$ are a function of $x$ (or of $T$ when $x$ is constant). Simultaneous growth of $J_{0}$ and $\Delta J$ with $x$ explains why disorder phenomena, such as critical nonlinear susceptibility and hierarchical relaxation [27], occur under the same conditions as ordering phenomena, such as diverging linear susceptibility and elastic compliance as well as critical slowing down. It also calls for caution when comparing data with results of the SK theory which considers $J_{0}$ and $\Delta J$ temperature-independent and of infinite range. True dipolar interactions in real crystals and particularly in KNT do not belong into this category. They have dipolar character only for large distances $r>r_{\mathrm{c}}$, whereas for $r<r_{\mathrm{c}}$ there are strong deviations : selfconsistent model calculation suggests that below $r_{\mathrm{c}}$ the randomness $\Delta J$ is considerably enhanced [8]. On the other hand, coupling between dipolar orientation and polar lattice modes adds an effective ferroelectric component [7] to $J_{0}$. Thus, the growth of $r_{\mathrm{c}}$ near $T_{\mathrm{c}}$ simultaneously drives the growth of both $J_{0}$ and $\Delta J$, the parameters which govern linear and nonlinear susceptibility, respectively. The result of a model calculation of dipolar interactions might render this suggestion less speculative.

\section{Conclusions.}

The linear susceptibility and the elastic compliance are critical near a temperature $T_{\mathrm{c}}$ which depends on the $\mathrm{Na}$ concentration of $(\mathrm{K}-\mathrm{Na}) \mathrm{TaO}_{3}$. They are monodispersive in the same frequency region; this indicates that a quadrupolar deformation follows the polar displacement of $\mathrm{Ta}$ adiabatically. Below $T_{\mathrm{c}}$, the linear susceptibility has a constant value of $3 \times 10^{4}$ from which we deduce a correlation volume of the polar displacement of $1.5 \times 10^{5}$ unit cells. This corresponds to $200 \AA$ linear dimension. Finite correlation, critical nonlinear susceptibility, and hierarchical relaxation are trademarks of a transition to a glassy state. They involve disordered polar motion attributed to off-center random-site $\mathrm{Na}$ ions.

Ordered motion of $\mathrm{Ta}$ and disordered motion are strikingly correlated. They occur in samples with $\mathrm{Na}$ concentration $x \geqslant 0.12$ and, there, in the neighborhood of the $x$-dependent critical temperature. We argue that the enhancement of the ferroelectric-type interaction between $\mathrm{Ta}$ ions at lattice sites is due to the volume gain for the polarizable oxygen ion arising from substitution of $\mathrm{K}^{+}$by the small $\mathrm{Na}^{+}$ion. Enhanced ferroelectric interaction entails enhanced polarizability of the lattice, and this triggers an increase of the interaction between impurity $\mathrm{Na}$ dipoles. This interaction is frustrated, i.e., it can be attractive or repulsive according to the ion pair considered. It is this enhanced frustrated interaction that ultimately leads to the formation of a dipolar glass state. This state is characterized by hierarchical relaxation evidenced by arithmetic dispersion in the low-frequency range, and a diverging nonlinear static susceptibility.

\section{References}

[1] Landolt-Börnstein, Numerical Data and Functional Relationships in Science and Technology, Ed. K. H. Hellwege (Springer-Verlag, Heidelberg) 16a 1981, $21 \mathrm{f}$.

[2] Lines, M. E. and Glass, A. M., Principles and Applications of Ferroelectrics and Related Materials (Clarendon, Oxford) 1977, p. 241.

[3] Burfoot, J. C. and TAYlor, G. W., Polar Dielectrics and Their Applications (Macmillan, London) 1979 , p. 13

[4] VAn DeR Klink, J. J., Rytz, D., Borsa, F. and Höchli, U. T., Phys. Rev. B 27 (1983) 89.
[5] Maglione, M., Grymaszewski, E. and Godefroy, G., to be published.

[6] p. 373 in [2].

[7] Vugmeister, B. E. and Glinchuk, M. D., Sov. Phys. JETP 52 (1980) 3.

[8] Wang, J. C., Phys. Rev. B 22 (1980) 2725.

[9] Davis, T. G., Phys. Rev. B 5 (1972) 2530.

[10] Rytz, D. Höchli, U. T. and Bilz, H., Phys. Rev. B 22 (1980) 359.

[11] Höchli, U. T. and Boatner, L. A., Phys. Rev. B 20 (1979) 266. 
[12] RYTZ, D. and BufFAT, D., private communication ; Buffat, P., Ganière, J. D., Rappaz, M. and RYTZ, D., J. Cryst. Growth 74 (1986) 353.

[13] Lanzi, G., Milani, P., Samoggia, G., Maglione, M. and Höchli, U. T., Phys. Rev. B 36 (1987) 1233.

[14] VAN DER KLINK, J. J. and RYTZ, D., Phys. Rev. B 27 (1983) 4471.

[15] Maglione, M., Höchli, U. T. and Joffrin, J., Phys. Rev. Lett. 57 (1986) 436.

[16] Maglione, M., Rod, S. and Höchli, U. T., Europhys. Lett. 4 (1987) 631.

[17] Binder, K. and Young, A. P., Rev. Mod. Phys. 58 (1986) 801, review analogous investigations on spin glasses. They raise the question whether the neglect of low-frequency dispersion had altered the conclusion as to the static nature of the spinglass transition, p. 828.

[18] Pappa, C., Hamman, J. and Jacobini, C., J. Phys. France 46 (1985) 637 ;

See also Omary, R., PréJean, J. J. and Souletie, J., for a slightly different approach, J. Phys. France 44 (1983) 1069.

[19] LeVY, L. P. and OGIELSKI, A. T., report nonlinear dynamic susceptibility in a spin glass, Phys. Rev. Lett. 57 (1986) 3288.
[20] Rupprecht, G. and Winter, W. H., Phys. Rev. 155 (1967) 1019.

[21] Borsa, F., HÖChli, U. T., VAN DER KLINK, J. J. and RYTZ, D., Phys. Rev. Lett. 45 (1980) 1884.

[22] Morf, R., Schneider, T. and Stoll, E., Phys. Rev. B 16 (1977) 462.

[23] Schmeltzer, D., Phys. Rev. B 28 (1983) 495.

[24] Sherrington, D. and Kirkpatrick, S., Phys. Rev. Lett. 35 (1975) 1792.

[25] Yacoby, Y., Solid State Commun. 15 (1974) 715.

[26] Höchli, U. T. and Rigamonti, A., J. Phys. C 16 (1983) 6321.

[27] Sompolinski, H. and Zippelius, A., Phys. Rev. B 25 (1983) 6860.

[28] TURSKI, A., private communication.

[29] Fossheim, K. and Fossum, J. O., in Multicritical Phenomena, Eds. R. Pynn and A. Skjeltorp (Plenum, New York) NATO ASI Ser. B 106 (1980) 113.

[30] Höchli, U. T. and Bruce, A. D., J. Phys. C 13 (1980) 1963.

[31] Hertz, J. A., Khurna, A. and Klemm, R. A., Phys. Rev. Lett. 66 (1981) 496.

[32] Migoni, R., Bilz, H. and BÄUerle, D., Phys. Rev. Lett. 37 (1976) 1155. 